\title{
THE HANKEL TRANSFORMATION OF BANACH-SPACE-VALUED GENERALIZED FUNCTIONS
}

\author{
E. L. KOH AND C. K. LI
}

(Communicated by Palle E. T. Jorgensen)

\begin{abstract}
The object of this paper is to study Banach-space-valued generalized functions belonging to $\left[H_{\mu}(A) ; B\right]$ for which the Hankel transformation may be defined. In Realizability theory for continuous linear systems (Academic Press, New York, 1972), Zemanian considered certain $\rho$-type testing function spaces for which the Laplace transformation is defined. Tiwari (Banach space valued distributional Mellin transform and form invariant linear filtering, Indian J. Pure Appl. Math. 20 (1989), 493-504) follows Zemanian in extending the Mellin transform. Their works are based on the denseness of the Schwartz space $D^{m}(A)$ in the testing function spaces of interest. This method is not possible here since the space $D^{m}(A)$ is not dense in $H_{\mu}(A)$, and the structure of $H_{\mu}(A)$ is quite different from that of $D^{m}(A)$, which has an inductive-limit topology. Thus, it is necessary to introduce a dense subspace ${ }_{\mu} D_{I}(A)$ of $H_{\mu}(A)$ to derive some properties of $H_{\mu}(A)$. We then define the Hankel transformation on $\left[H_{\mu}(A) ; B\right]$. We end this paper with some operational formulas, which are analogous with those given by the first author in SIAM J. Math. Anal. 1 (1970), 322-327.
\end{abstract}

\section{INTRODUCTION}

Zemanian studied the theory of Banach-space-valued testing functions and distributions in [2], which is somewhat more general than that of scalar distributions. He constructed $D^{m}(A)$ as the inductive-limit space given by

$$
D^{m}(A)=D_{R^{n}}^{m}(A)=\bigcup_{j=1}^{\infty} D_{K_{j}}^{m}(A)
$$

where $D_{K_{j}}^{m}(A)$ denotes the linear space of all smooth functions $\phi$ from $R^{n}$ into a Banach space $A$ such that $\operatorname{supp} \varphi \subset K_{j} . K_{j}$ are compact subsets of $R^{n}$ and $K_{j} \subset K_{j+1}, \bigcup_{j=1}^{\infty} K_{j}=R^{n}$. We assign to $D_{K_{j}}^{m}(A)$ the topology generated by the collection $\left\{\gamma_{k} ; 0 \leq k \leq m\right\}$ of seminorms, where $\gamma_{k}(\phi) \triangleq \sup _{t \in K_{j}}\left\|\phi^{(k)}(t)\right\|_{A}$.

Applying the interpolation theory, he describes the local structure property below.

Received by the editors January 21, 1992; this paper was presented on December 10,1991, at the winter meeting of the Canadian Mathematical Society in Victoria, Canada.

1991 Mathematics Subject Classification. Primary 46F10.

Key words and phrases. The Hankel transformation, inductive-limit topology, generalized functions, Banach space. 
Theorem 1.1. Let $f \in\left[D^{m}(A) ; B\right]$ and $K$ be a compact interval in $R^{n}$. Then there exists an integer $p \in R^{n}$ with $0 \leq p \leq m$ and a continuous $[A ; B]$-valued function $h$ on $K$ such that, for all $\phi \in D_{K}^{m+[2]}(A)$,

$$
\langle f, \phi\rangle=\int_{K} h(t) D^{p+[2]} \phi(t) d t .
$$

In general, $p$ and $h$ depend on $f$ and $K$.

Finally, he mentions

Theorem 1.2. If $\mathscr{T}^{m}$ and $\mathscr{T}^{m}(A)$ are normal spaces (i.e., $D$ is dense in $\mathscr{T}^{m}$ and $\left.\mathscr{T}^{m}(A)\right)$, then there exists a bijection from $\left[\mathscr{T}^{m}(A) ; B\right]$ onto $\left[\mathscr{T}^{m} ;[A ; B]\right]$ defined by

$$
\langle g, \psi\rangle a \triangleq\langle f, \psi a\rangle, \quad \psi \in \mathscr{T}^{m}, a \in A,
$$

where $g \in\left[\mathscr{T}^{m} ;[A ; B]\right], f \in\left[\mathscr{T}^{m}(A) ; B\right]$.

Tiwari [3] mimics the method of Zemanian in defining Banach-space-valued distributions for which a Mellin transform can be given. Several properties including a Mellin-type convolution theorem are proved. These results are similar to those of Zemanian [1].

In this paper, we introduce a dense subspace ${ }_{\mu} D_{I}(A)$ of $H_{\mu}(A)$. It does not have an inductive-limit topology. The local structure theorem is no longer discussed in $\left[H_{\mu}(A) ; B\right]$. However, with a different method, we show that there is still a bijection from $\left[H_{\mu}(A) ; B\right]$ onto $\left[H_{\mu} ;[A ; B]\right]$. Further, we are able to define the Hankel transformation of arbitrary order on $H_{\mu}(A)$, which is still an automorphism on $H_{\mu}(A)$. We give some operational formulas at the end of this paper.

Our notation is similar to that used in [1, 2, 4]. Given any two topological vector spaces $A$ and $B,[A ; B]$ denotes the linear space of all continuous linear mappings of $A$ into $B$. The element of $B$ assigned by $f \in[A ; B]$ to $\phi \in A$ is denoted by $\langle f, \phi\rangle .[A ; B]$ is supplied with the topology of uniform convergence on bounded sets in $A$. $\|\cdot\|_{B}$ denotes the norm in any Banach space $B . R$ and $C$ are the real and complex number fields. $I$ is the open interval $(0,+\infty)$. Other notation will be introduced as the need arises.

$$
\begin{aligned}
& \text { 2. THE CORRESPONDENCE BETWEEN }\left[H_{\mu}(A) ; B\right] \\
& \text { AND }\left[H_{\mu} ;[A ; B]\right] \text { FOR } \mu \geq-\frac{1}{2}
\end{aligned}
$$

Following Zemanian, $H_{\mu}(A)$ is defined as follows:

Definition 2.1. Let $x$ be a real variable restricted to $I$. For each real number $\mu, \phi(x) \in H_{\mu}(A)$ iff it is defined on $I$, takes its values in $A$, is smooth, and for each pair of nonnegative integers $m$ and $k$

$$
\gamma_{m, k}^{\mu}(\phi) \triangleq \sup _{x \in I}\left\|x^{m}\left(x^{-1} D\right)^{k} x^{-\mu-1 / 2} \phi(x)\right\|_{A}
$$

is finite. $H_{\mu}(A)$ is a linear space. The topology of $H_{\mu}(A)$ is that generated by $\left\{\gamma_{m, k}^{\mu}\right\}_{m, k=0}^{\infty}$.

Definition 2.2. $\phi(x) \in D_{I}(A)$ iff $\phi$ is defined on $I$, takes its value in $A$, is smooth, and for every $\phi$ there exists $b \in I$ such that $\phi(x)=0$ for $x \in$ $[b,+\infty)$. Let ${ }_{\mu} D_{I}(A) \triangleq D_{I}(A) \cap H_{\mu}(A)$. 
Theorem 2.1. The subspace ${ }_{\mu} D_{I}(A)$ is dense in $H_{\mu}(A)$ for all $\mu \in R$.

Proof. Let $\lambda(x) \in D_{I}(C)$ such that $\lambda(x)=1$ for $0<x \leq 1$ and $\lambda(x)=0$ for $x \geq 2$.

For arbitrary $\phi(x) \in H_{\mu}(A)$ and each pair of nonnegative integers $m$ and $k$ we consider

$$
\begin{aligned}
& x^{m}\left(x^{-1} D\right)^{k} x^{-\mu-1 / 2}[\lambda(x / N) \phi(x)-\phi(x)] \\
& \quad=x^{m+1} \sum_{v=0}^{k}\left(\begin{array}{l}
k \\
v
\end{array}\right)\left(x^{-1} D\right)^{k-v} x^{-\mu-1 / 2} \phi \frac{\left(x^{-1} D\right)^{v}[\lambda(x / N)-1]}{x} .
\end{aligned}
$$

Therefore,

$$
\begin{aligned}
& \sup _{x \in I}\left\|x^{m}\left(x^{-1} D\right)^{k} x^{-\mu-1 / 2}(\lambda(x / N) \phi(x)-\phi(x))\right\|_{A} \\
& \quad \leq \sum_{v=0}^{k}\left(\begin{array}{l}
k \\
v
\end{array}\right) \sup _{x \in I}\left\|x^{m+1}\left(x^{-1} D\right)^{k-v} x^{-\mu-1 / 2} \phi\right\|_{A} \cdot \sup _{x \geq N}\left|\frac{\left(x^{-1} D\right)^{v}[\lambda(x / N)-1]}{x}\right| .
\end{aligned}
$$

It follows from $\phi \in H_{\mu}(A)$ that

$$
\sup _{x \in I}\left\|\left(x^{-1} D\right)^{k-v} x^{-\mu-1 / 2} \phi\right\|_{A}
$$

is finite.

Since $\lambda(x)$ and its derivatives are bounded, it follows that

$$
\sup _{x \geq N}\left|\frac{\left(x^{-1} D\right)^{v}[\lambda(x / N)-1]}{x}\right| \rightarrow 0 \text { as } N \rightarrow \infty \text {, for fixed } k \text { and } 0 \leq v \leq k \text {, }
$$

whence our assertion.

$H_{\mu}(A)$ is not a $\rho$-type testing function space in the sense of Zemanian [2]. To see this, we choose $\phi(x)=x^{\mu+1 / 2} e^{-x^{2}} a_{0}, a_{0} \in A$ and $a_{0} \neq 0$. Then for all $\psi$, which is smooth from $I$ into $A$ with compact support contained in $I$, $\gamma_{0,0}^{\mu}(\phi-\psi) \geq\left\|a_{0}\right\| / 2>0$. This means the balloon

$$
\left\{\theta ; \theta \in H_{\mu}(A), \gamma_{0,0}^{\mu}(\phi-\theta) \leq\left\|a_{0}\right\| / 3\right\}
$$

does not contain any element of $D^{m}(A)$. Thus our result is true.

The following lemmas will be used subsequently (see [1, 2]).

Lemma 2.1. Let $V, W$ be locally convex spaces, and let $\Gamma$ and $P$ generate families of seminorms of the topologies of $V$ and $W$, respectively. Let $f$ be a linear mapping of $V$ into $W$. The following four assertions are equivalent:

(i) $f$ is continuous.

(ii) $f$ is continuous at the origin.

(iii) For every continuous seminorm $\rho$ on $W$, there exists a continuous seminorm $\gamma$ on $V$ such that $\rho(f(\theta)) \leq \gamma(\theta)$ for all $\theta$.

(iv) For every $\rho \in P$, there exists a constant $M>0$ and a finite collection $\left\{\gamma_{1}, \gamma_{2}, \ldots, \gamma_{m}\right\} \subset \Gamma$ such that

$$
\rho(f(\theta)) \leq M \max _{0 \leq k \leq m} \gamma_{k}(\theta)
$$

for all $\theta \in V$.

Lemma 2.2. For $\mu \geq-\frac{1}{2}$, the conventional $h_{\mu}$ is an automorphism on $H_{\mu}(A)$. Proof. Very similar to Theorem 5.4.-1, p. 141 of [1]. 
Theorem 2.2. Every $f \in\left[H_{\mu}(A) ; B\right]$ uniquely defines a $g \in\left[H_{\mu} ;[A ; B]\right]$ through the equation

$$
\langle g, \theta\rangle a \triangleq\langle f, \theta a\rangle, \quad \theta \in H_{\mu}, a \in A,
$$

for all $\mu \in R$.

Proof. Fixing upon some $\theta \in H_{\mu}$ we define a mapping $j_{\theta}$ of $A$ into $B$ by $j_{\theta} a=\langle f, \theta a\rangle$ for all $a \in A$. It readily follows that $j_{\theta}$ is linear. By Lemma 2.1 (iv) there exist positive integers $m_{0}, k_{0}$ and a constant $M>0$ such that

$$
\left\|j_{\theta} a\right\|_{B}=\|\langle f, \theta a\rangle\|_{B} \leq M \max _{\substack{0 \leq k \leq k_{0} \\ 0 \leq m \leq m_{0}}} \gamma_{m, k}^{\mu}(\theta a)
$$

where

$$
\begin{aligned}
\gamma_{m, k}^{\mu}(\theta a) & =\sup _{x \in I}\left\|x^{m}\left(x^{-1} D\right)^{k} x^{-\mu-1 / 2} \theta a\right\|_{A} \\
& =\|a\|_{A} \sup _{x \in I}\left|x^{m}\left(x^{-1} D\right)^{k} x^{-\mu-1 / 2} \theta\right| .
\end{aligned}
$$

Hence

$$
\left\|j_{\theta} a\right\|_{B} \leq M\|a\|_{A} \max _{\substack{0 \leq k \leq k_{0} \\ 0 \leq m \leq m_{0}}} \gamma_{m, k}^{\mu}(\theta)
$$

and

$$
\left\|j_{\theta}\right\|_{[A ; B]} \leq M \max _{\substack{0 \leq k \leq k_{0} \\ 0 \leq m \leq m_{0}}} \gamma_{m, k}^{\mu}(\theta) .
$$

Next, set $\langle g, \theta\rangle \triangleq j_{\theta}$. This uniquely defines $g$ as a mapping from $H_{\mu}$ into $[A ; B] . g$ is linear because, for any $a \in A, \alpha, \beta \in C$, and $\theta, \psi \in H_{\mu}$

$$
\begin{aligned}
\langle g, \alpha \theta+\beta \psi\rangle a & =\langle f, \alpha \theta a+\beta \psi a\rangle=\alpha\langle f, \theta a\rangle+\beta\langle f, \psi a\rangle \\
& =(\alpha\langle g, \theta\rangle+\beta\langle g, \psi\rangle) a .
\end{aligned}
$$

Moreover, (2.1) implies that $g$ is continuous.

Let ${ }_{\mu} D_{I} \odot A$ denote the linear space of all $\varphi \in{ }_{\mu} D_{I}(A)$ having representations of the form $\varphi=\sum \theta_{k} a_{k}$ where $\theta_{k} \in{ }_{\mu} D_{I}, a_{k} \in A$, and the summation is over a finite number of terms.

Theorem 2.3. The space ${ }_{\mu} D_{I} \odot A$ is dense in $H_{\mu}(A)$ for $\mu \geq-\frac{1}{2}$.

Proof. Let $\lambda(x)$ be defined as in the proof of Theorem 2.1. For $\varphi \in{ }_{\mu} D_{I}(A)$, we first show that

$$
\lambda(x / N) h_{\mu}(\varphi) \rightarrow h_{\mu}(\varphi) \text { in } H_{\mu}(A) \text { as } N \rightarrow+\infty \text { for all } \mu \in R .
$$

The following equation will be used (see [1]):

$$
\begin{aligned}
& (-1)^{m+k} y^{m}\left(y^{-1} D\right)^{k} y^{-\mu-1 / 2} h_{\mu}(\varphi)(y) \\
& \quad=\int_{0}^{+\infty} x^{2 \mu+2 k+m+1}\left[\left(x^{-1} D\right)^{m} x^{-\mu-1 / 2} \varphi(x)\right] \frac{J_{\mu+k+m}(x y)}{(x y)^{\mu+k}} d x .
\end{aligned}
$$

Hence

$$
\begin{aligned}
& \sup _{x \in I}\left\|x^{m}\left(x^{-1} D\right)^{k} x^{-\mu-1 / 2} h_{\mu}(\varphi)\left[\lambda\left(\frac{x}{N}\right)-1\right]\right\|_{A} \\
& \leq \sum_{v=0}^{k}\left(\begin{array}{l}
k \\
v
\end{array}\right) \sup _{x \geq N}\left|\frac{\left(x^{-1} D\right)^{v}[\lambda(x / N)-1]}{x}\right| \sup _{x \in I}\left\|x^{m+1}\left(x^{-1} D\right)^{k-v} x^{-\mu-1 / 2} h_{\mu}(\varphi)\right\|_{A} .
\end{aligned}
$$


By what we have proved in Theorem 2.1,

$$
\sup _{x \geq N}\left|\frac{\left(x^{-1} D\right)^{v}[\lambda(x / N)-1]}{x}\right| \rightarrow 0 \text { as } N \rightarrow \infty \text { for fixed } k \text { and } 0 \leq v \leq k .
$$

Using equation (2.2) and noting that $J_{\mu+k-v+m+1}(x y) /(x y)^{\mu+k-v}$ is bounded, say by $B_{k, v, m}$, we get

$$
\begin{aligned}
& \sup _{x \in I}\left\|x^{m+1}\left(x^{-1} D\right)^{k-v} x^{-\mu-1 / 2} h_{\mu}(\varphi)\right\|_{A} \\
& \quad=\sup _{x \in I}\left\|\int_{0}^{+\infty} y^{2 \mu+2(k-v)+m+2}\left[\left(y^{-1} D\right)^{m+1} y^{-\mu-1 / 2} \varphi(y)\right] \frac{J_{\mu+k-v+m+1}(x y)}{(x y)^{\mu+k-v}} d y\right\|_{A} .
\end{aligned}
$$

Choosing a positive integer $n$ such that

$$
y^{2 \mu+2(k-v)+m+2} \leq\left(1+y^{2}\right)^{n} \quad \text { for all } y \in I
$$

we have

$$
\begin{gathered}
\sup _{y \in I}\left\|y^{2 \mu+2(k-v)+m+2}\left[\left(y^{-1} D\right)^{m+1} y^{-\mu-1 / 2} \varphi(y)\right]\right\|_{A} \\
\leq \sup _{y \in I}\left\|\left(1+y^{2}\right)^{n}\left[\left(y^{-1} D\right)^{m+1} y^{-\mu-1 / 2} \varphi(y)\right]\right\|_{A} .
\end{gathered}
$$

Since $\varphi \in{ }_{\mu} D_{I}(A)$, there exists $b \in I$ such that $\varphi(x)=0$ for $x \in[b,+\infty)$. It follows that

$$
\begin{aligned}
& \sup _{x \in I}\left\|x^{m+1}\left(x^{-1} D\right)^{k-v} x^{-\mu-1 / 2} h_{\mu}(\varphi)\right\|_{A} \\
& \quad \leq B_{k, v, m} b \sup _{y \in I}\left\|\left(1+y^{2}\right)^{n}\left[\left(y^{-1} D\right)^{m+1} y^{-\mu-1 / 2} \varphi(y)\right]\right\|_{A}
\end{aligned}
$$

is finite. Therefore,

$$
\lambda(x / N) h_{\mu}(\varphi) \rightarrow h_{\mu}(\varphi) \text { in } H_{\mu}(A) \text { as } N \rightarrow+\infty .
$$

Second, we prove that ${ }_{\mu} D_{I} \odot A$ is dense in $H_{\mu}(A)$ for $\mu \geq-\frac{1}{2}$. For a positive integer $m_{1}$, we have

$$
\sqrt{x y} J_{\mu}(x y)=\sum_{j=0}^{m_{1}} \frac{(x y)^{1 / 2}(-1)^{j}(x y / 2)^{\mu+2 j}}{j ! \Gamma(\mu+j+1)}+\sum_{j=m_{1}+1}^{\infty} \frac{(x y)^{1 / 2}(-1)^{j}(x y / 2)^{\mu+2 j}}{j ! \Gamma(\mu+j+1)} .
$$

For every $\varphi \in{ }_{\mu} D_{I}(A)$, the term

$$
T_{N, m_{1}}=\lambda\left(\frac{x}{N}\right) \int_{0}^{+\infty} \varphi(t) \sum_{j=0}^{m_{1}} \frac{(x t)^{1 / 2}(-1)^{j}(x t / 2)^{\mu+2 j}}{j ! \Gamma(\mu+j+1)} d t
$$

where $N, m_{1}=1,2, \ldots$, belongs to ${ }_{\mu} D_{I} \odot A$ since $\mu \geq-\frac{1}{2}$. Now

$$
\begin{aligned}
T_{N, m_{1}} & -\int_{0}^{+\infty} \varphi(t) \sqrt{x t} J_{\mu}(x t) d t \\
= & T_{N, m_{1}}-\lambda\left(\frac{x}{N}\right) \int_{0}^{+\infty} \varphi(t) \sqrt{x t} J_{\mu}(x t) d t \\
& +\lambda\left(\frac{x}{N}\right) \int_{0}^{+\infty} \varphi(t) \sqrt{x t} J_{\mu}(x t) d t-\int_{0}^{+\infty} \varphi(t) \sqrt{x t} J_{\mu}(x t) d t
\end{aligned}
$$


By what we have just proved, for arbitrary $\varepsilon>0$, there exists an $N_{1}$ such that for $N \geq N_{1}$, we have

$$
\sup _{x \in I}\left\|x^{m}\left(x^{-1} D\right)^{k} x^{-\mu-1 / 2}\left[\lambda(x / N) h_{\mu}(\varphi)-h_{\mu}(\varphi)\right]\right\|_{A}<\varepsilon / 2 .
$$

Fixing $N\left(\geq N_{1}\right)$, then

$$
\lambda\left(\frac{x}{N}\right)\left[\sum_{j=0}^{m_{1}} \frac{(x t)^{1 / 2}(-1)^{j}(x t / 2)^{\mu+2 j}}{j ! \Gamma(\mu+j+1)}-\sqrt{x t} J_{\mu}(x t)\right]
$$

and its derivatives with respect to $x$ converge to zero uniformly on every compact subset of $I$. It has a uniformly bounded support. Therefore it converges in the sense of Schwartz, whose topology is stronger than that of $H_{\mu}$ (see [1]). It follows that there exists an $L \in I$ such that as long as $m_{1} \geq L$, then for all $t \leq b$,

$$
\begin{aligned}
\sup _{x \in I} & \left|x^{m}\left(x^{-1} D\right)^{k} x^{-\mu-1 / 2} \lambda\left(\frac{x}{N}\right)\left[\sum_{j=0}^{m_{1}} \frac{(x t)^{1 / 2}(-1)^{j}(x t / 2)^{\mu+2 j}}{j ! \Gamma(\mu+j+1)}-\sqrt{x t} J_{\mu}(x t)\right]\right| \\
& \leq \frac{\varepsilon}{2 M_{1}},
\end{aligned}
$$

where $M_{1}=b \sup _{t \in I}\|\varphi(t)\|_{A}$. If $M_{1}=0$, then there is nothing to be proved. Therefore,

$$
\sup _{x \in I}\left\|x^{m}\left(x^{-1} D\right)^{k} x^{-\mu-1 / 2}\left[T_{N, m_{1}}-\int_{0}^{+\infty} \varphi(t) \sqrt{x t} J_{\mu}(x t) d t\right]\right\|_{A}<\varepsilon
$$

provided $N \geq N_{1}, m_{1} \geq L$.

Since $h_{\mu}$ is an automorphism on $H_{\mu}(A)$ for $\mu \geq-\frac{1}{2}$ by Lemma 2.2 and ${ }_{\mu} D_{I}(A)$ is dense in $H_{\mu}(A)$, it follows that $h_{\mu}\left({ }_{\mu} D_{I}(A)\right)$ is dense in $H_{\mu}(A)$. Our assertion follows directly from the fact that ${ }_{\mu} D_{I} \odot A$ is dense in $h_{\mu}\left({ }_{\mu} D_{I}(A)\right)$.

Theorem 2.4. There is a bijection from $\left[H_{\mu}(A) ; B\right]$ onto $\left[H_{\mu} ;[A ; B]\right]$ defined by

$$
\langle g, \theta\rangle a=\langle f, \theta a\rangle
$$

where $a \in A, g \in\left[H_{\mu} ;[A ; B]\right]$, and $f \in\left[H_{\mu}(A) ; B\right], \theta \in H_{\mu}$ for $\mu \geq-\frac{1}{2}$.

Proof. By Theorem 2.2, every $f \in\left[H_{\mu}(A) ; B\right]$ uniquely defines a $g \in$ $\left[H_{\mu} ;[A ; B]\right]$ through the equation

$$
\langle g, \theta\rangle a \triangleq\langle f, \theta a\rangle \text { for all } \mu \in R
$$

Let us consider the converse. For every $\varphi \in{ }_{\mu} D_{I} \odot A$, we define

$$
\langle f, \varphi\rangle=\sum\left\langle g, \theta_{k}\right\rangle a_{k} \text { for } \varphi=\sum \theta_{k} a_{k} .
$$

It follows from the definition that $f$ is linear on ${ }_{\mu} D_{I} \odot A$. We wish to show that $f$ is continuous. Indeed, for arbitrary $\varepsilon>0$, as long as $\theta a \quad\left(\theta \in{ }_{\mu} D_{I}\right.$, $a \in A$ ) belongs to the balloon $\left\{\varphi ; \gamma_{m, k}^{\mu}(\varphi)<\varepsilon / M, m=0,1, \ldots, m_{0}\right.$, $\left.k=0,1, \ldots, k_{0}\right\} . M, m_{0}, k_{0}$ are defined as follows. We infer that

$$
\|\langle f, \theta a\rangle\|_{B}=\|\langle g, \theta\rangle a\|_{B} \leq\|a\|_{A} \cdot\|\langle g, \theta\rangle\|_{[A ; B]} .
$$


By Lemma 2.1(iv), there exist $M>0$ and positive integers $m_{0}, k_{0}$ such that

$$
\|\langle f, \theta a\rangle\|_{B} \leq\|a\|_{A} \cdot M \max _{\substack{0 \leq k \leq k_{0} \\ 0 \leq m \leq m_{0}}} \gamma_{m, k}^{\mu}(\theta)<M \cdot \varepsilon / M=\varepsilon .
$$

Therefore $f$ is continuous at the origin. By Lemma 2.1(ii), $f$ is continuous on ${ }_{\mu} D_{I} \odot A$. According to Theorem 2.3, ${ }_{\mu} D_{I} \odot A$ is dense in $H_{\mu}(A)$ for $\mu \geq-\frac{1}{2}$. Thus our assertion is true.

3. The HANKel tRANSFORMATION $h_{\mu}$ ON $H_{\mu}(A)$ AND THE CORRESPONDENCE BETWEEN $\left[H_{\mu}(A) ; B\right]$ AND $\left[H_{\mu} ;[A ; B]\right]$ FOR ARBITRARY $\mu$

We shall use the following differential and integral operators due to Zemanian [1]:

$$
\begin{gathered}
N_{\mu} \varphi(x) \triangleq x^{\mu+1 / 2} D x^{-\mu-1 / 2} \varphi(x), \\
M_{\mu} \varphi(x) \triangleq x^{-\mu-1 / 2} D x^{\mu+1 / 2} \varphi(x), \\
N_{\mu}^{-1} \varphi(x) \triangleq x^{\mu+1 / 2} \int_{\infty}^{x} t^{-\mu-1 / 2} \varphi(t) d t .
\end{gathered}
$$

Lemma 3.1. $N_{\mu}$ is a continuous linear mapping of $H_{\mu}(A)$ into $H_{\mu+1}(A)$.

Indeed, $\gamma_{m, k}^{\mu+1}\left(N_{\mu} \varphi\right)=\gamma_{m, k+1}^{u}(\varphi)$ for every $\varphi \in H_{\mu}(A)$ and every choice of $m$ and $k$.

Lemma 3.2. $N_{\mu}^{-1}$ is a continuous linear mapping of $H_{\mu+1}(A)$ into $H_{\mu}(A)$.

Proof. Assume that $\varphi(x) \in H_{\mu+1}(A)$ and $k$ is a fixed positive integer. Then

$$
\begin{aligned}
\left(x^{-1} D\right)^{k} x^{-\mu-1 / 2} N_{\mu}^{-1} \varphi(x) & =\left(x^{-1} D\right)^{k} x^{-\mu-1 / 2} x^{\mu+1 / 2} \int_{\infty}^{x} t^{-\mu-1 / 2} \varphi(t) d t \\
& =\left(x^{-1} D\right)^{k-1} x^{-\mu-3 / 2} \varphi(x) .
\end{aligned}
$$

Hence

$$
\gamma_{m, k}^{\mu}\left(N_{\mu}^{-1} \varphi\right)=\gamma_{m, k-1}^{\mu+1}(\varphi), \quad k=1,2,3, \ldots, m=0,1,2, \ldots
$$

A similar result for the case $k=0$ can be derived as follows:

$$
\begin{aligned}
\left\|x^{m} x^{-\mu-1 / 2} N_{\mu}^{-1} \varphi(x)\right\|_{A} & \leq x^{m} \int_{x}^{\infty}\left\|t^{-\mu-1 / 2} \varphi(t)\right\|_{A} d t \\
& \leq \int_{x}^{\infty}\left\|t^{m} t^{-\mu-1 / 2} \varphi(t)\right\|_{A} d t \\
& \leq \int_{0}^{+\infty}\left\|\frac{1}{1+t^{2}}\left(t^{m+1}+t^{m+2}\right) t^{-\mu-3 / 2} \varphi(t)\right\|_{A} d t \\
& \leq \int_{0}^{\infty} \frac{1 d t}{1+t^{2}} \cdot \sup _{t \in I}\left\|\left(t^{m+1}+t^{m+3}\right) t^{-\mu-3 / 2} \varphi(t)\right\|_{A} .
\end{aligned}
$$

Therefore

$$
\gamma_{m, 0}^{\mu}\left(N_{\mu}^{-1} \varphi\right) \leq \frac{\pi}{2}\left[\gamma_{m+1,0}^{\mu+1}(\varphi)+\gamma_{m+3,0}^{\mu+1}(\varphi)\right], \quad m=0,1,2, \ldots
$$

It follows from the above that $N_{\mu}^{-1}$ is a continuous linear mapping of $H_{\mu+1}(A)$ into $H_{\mu}(A)$. 
Let $\mu \in R$ and let $k$ be a positive integer such that $\mu+k \geq-\frac{1}{2}$. Assume that $\varphi \in H_{\mu}(A)$ and define $h_{\mu, k}$ on $H_{\mu}(A)$ by (see Koh [4])

$$
\Phi(x)=h_{\mu, k}(\phi(y)) \triangleq(-1)^{k} x^{-k} h_{\mu+k} N_{\mu+k-1} \cdots N_{\mu+1} N_{\mu} \varphi(y) .
$$

Let $\Phi(x) \in H_{\mu}(A)$ and define $h_{\mu, k}^{-1}$ on $H_{\mu}(A)$ by

$$
\varphi(y)=h_{\mu, k}^{-1}(\Phi(x)) \triangleq(-1)^{k} N_{\mu}^{-1} N_{\mu+1}^{-1} \cdots N_{\mu+k-1}^{-1} h_{\mu+k} x^{k} \Phi(x) .
$$

Theorem 3.1. $h_{\mu, k}$ is an automorphism on $H_{\mu}(A)$. Its inverse is $h_{\mu, k}^{-1}$ and $h_{\mu, k}=h_{\mu}$ if $\mu \geq-\frac{1}{2}$.

Proof. By Lemmas 3.1 and 3.2, $\varphi \rightarrow N_{\mu} N_{\mu+1} \cdots N_{\mu+k-1} \varphi$ is an isomorphism from $H_{\mu}(A)$ onto $H_{\mu+k}(A)$.

By Lemma 2.2, $h_{\mu+k}$ is an automorphism on $H_{\mu+k}(A)$ for $\mu+k \geq-\frac{1}{2}$. It follows from $\gamma_{m, k}^{\mu}\left(x^{-k} \varphi\right)=\gamma_{m, k}^{\mu+k}(\varphi)$ that $\varphi \rightarrow x^{-k} \varphi$ is an isomorphism from $H_{\mu+k}(A)$ onto $H_{\mu}(A)$. Therefore $h_{\mu, k}$ is an automorphism on $H_{\mu}(A)$. Similarly $h_{\mu, k}^{-1}$ is an automorphism on $H_{\mu}(A)$ and is inverse to $h_{\mu, k}$ because $h_{\mu+k}^{-1}=h_{\mu+k}$ and the inverse of $N_{\mu+k-1} \cdots N_{\mu}$ is $N_{\mu}^{-1} \cdots N_{\mu+k-1}^{-1}$.

To prove the last statement, let $\varphi(y) \in H_{\mu}(A), \mu \geq-\frac{1}{2}$, and consider $k=1$;

$$
\begin{aligned}
h_{\mu, 1} \varphi & =-x^{-1} h_{\mu+1} N_{\mu} \varphi=-x^{-1} \int_{0}^{\infty} y^{\mu+1 / 2}\left[D_{y} y^{-\mu-1 / 2} \varphi(y)\right] \sqrt{x y} J_{\mu+1}(x y) d y \\
& =-\left.x^{-1} \sqrt{x y} J_{\mu+1}(x y) \varphi(y)\right|_{0} ^{\infty}+\int_{0}^{\infty} \varphi(y) \sqrt{x y} J_{\mu}(x y) d y .
\end{aligned}
$$

Since $\varphi(y)$ is of rapid descent and $\sqrt{x y} J_{\mu+1}(x y)$ is bounded as $y \rightarrow \infty$, while $\varphi(y)=O\left(y^{\mu+1 / 2}\right)$ and $\sqrt{x y} J_{\mu+1}(x y)=O\left(y^{\mu+3 / 2}\right)$ as $y \rightarrow 0^{+}$, the limit terms are zero for $\mu \geq-\frac{1}{2}$. Thus $h_{\mu, 1} \varphi=h_{\mu} \varphi$. By induction, $h_{\mu, k}=h_{\mu}$ for $\mu \geq-\frac{1}{2}$.

Note that the definition of $h_{\mu, k}$ is independent of the choice of $k$ so long as $k+\mu \geq-\frac{1}{2}$. Indeed, if $k>p \geq-\mu-\frac{1}{2}$, then $h_{\mu+p, k-p}=h_{\mu+p}$ by Theorem 3.1; hence

$$
\begin{aligned}
h_{\mu, k} \varphi & =(-1)^{k} x^{-k} h_{\mu+k} N_{\mu+k-1} \cdots N_{\mu} \varphi \\
& =(-1)^{p} x^{-p}(-1)^{k-p} x^{-(k-p)} h_{\mu+p+k-p} N_{\mu+p+k-p-1} \cdots N_{\mu+p} N_{\mu+p-1} \cdots N_{\mu} \varphi \\
& =(-1)^{p} x^{-p} h_{\mu+p, k-p} N_{\mu+p-1} \cdots N_{\mu} \varphi \\
& =(-1)^{p} x^{-p} h_{\mu+p} N_{\mu+p-1} \cdots N_{\mu} \varphi=h_{\mu, p} \varphi .
\end{aligned}
$$

Definition 3.1. Let $\mu \in R$ and $k$ be a positive integer such that $\mu+k \geq-\frac{1}{2}$. For any $f \in\left[H_{\mu}(A) ; B\right]$, the generalized Hankel transform $h_{\mu}^{\prime} f$ is defined by

$$
\left\langle h_{\mu}^{\prime} f, \varphi\right\rangle=\left\langle f, h_{\mu, k} \varphi\right\rangle, \quad \varphi \in H_{\mu}(A) .
$$

By Theorem 3.1 and the fact that $h_{\mu}^{\prime}$ is the adjoint operator of $h_{\mu, k}$ on $H_{\mu}(A)$, we have

Theorem 3.2. $h_{\mu}^{\prime}$ is an automorphism on $\left[H_{\mu}(A) ; B\right]$ for all $\mu \in R$. 
Applying operator $T \triangleq N_{\mu+k-1} \cdots N_{\mu}$, we have

Theorem 3.3. Let $A$ and $B$ be two Banach spaces. There is a bijection from $\left[H_{\mu}(A) ; B\right]$ onto $\left[H_{\mu} ;[A ; B]\right]$ defined by

$$
\langle g, \theta\rangle a=\langle f, \theta a\rangle
$$

where $a \in A, \theta \in H_{\mu}, g \in\left[H_{\mu} ;[A ; B]\right]$, and $f \in\left[H_{\mu}(A) ; B\right], \mu \in R$.

Proof. For arbitrary $\mu \in R$, we choose a positive integer $k$ such that $\mu+k \geq$ $-\frac{1}{2}$. The operator $T$ is an isomorphism from ${ }_{\mu} D_{I} \odot A$ onto ${ }_{\mu+k} D_{I} \odot A$, which is dense in $H_{\mu+k}(A)$. Also $T$ is an isomorphism from $H_{\mu}(A)$ onto $H_{\mu+k}(A)$. Therefore, ${ }_{\mu} D_{I} \odot A$ is dense in $H_{\mu}(A)$. By Theorems 2.3 and 2.4, there is a bijection from $\left[H_{\mu}(A) ; B\right]$ onto $\left[H_{\mu} ;[A ; B]\right]$ satisfying the above equation.

\section{SOME OPERATIONAL FORMULAS}

We now establish certain transformation formulas relating to the Bessel-type differential operator $M_{\mu} N_{\mu}$, which are similar to those obtained in [4], but on $H_{\mu}(A)$.

Lemma 4.1. Let $\mu$ be a fuxed real number and $k$ a positive integer $\geq-\mu-\frac{1}{2}$. Then for every $\varphi \in H_{\mu}(A), h_{\mu+1, k}\left(N_{\mu} \varphi\right)=-x h_{\mu, k}(\varphi)$.

Proof. By definition

$$
\begin{aligned}
h_{\mu+1, k}\left(N_{\mu} \varphi\right) & =(-1)^{k} x^{-k} h_{\mu+1+k} N_{\mu+1+k} \cdots N_{\mu+1} N_{\mu} \varphi \\
& =-x h_{\mu, k+1}(\varphi)=-x h_{\mu, k}(\varphi) .
\end{aligned}
$$

Turning to the linear operator $M_{\mu}$, we prove that $\varphi \rightarrow M_{\mu} \varphi$ is a continuous linear mapping of $H_{\mu+1}(A)$ onto $H_{\mu}(A)$. Indeed, for $\varphi \in H_{\mu+1}(A)$ and any choice $m$ and $k$

$$
\begin{aligned}
\gamma_{m, k}^{\mu}\left(M_{\mu} \varphi\right)= & \sup _{x \in I}\left\|x^{m}\left(x^{-1} D\right)^{k} x^{-2 \mu-1} D x^{2 \mu+2} x^{-\mu-3 / 2} \varphi(x)\right\|_{A} \\
= & \sup _{x \in I} \|(2 \mu+2) x^{m}\left(x^{-1} D\right)^{k} x^{-\mu-3 / 2} \varphi(x) \\
& \quad+x^{m}\left(x^{-1} D\right)^{k} x^{2}\left(x^{-1} D\right) x^{-\mu-3 / 2} \varphi(x) \|_{A} \\
= & \cdots=\sup _{x \in I} \| 2(\mu+k+1) x^{m}\left(x^{-1} D\right)^{k} x^{-\mu-3 / 2} \varphi(x) \\
& +x^{m+2}\left(x^{-1} D\right)^{k+1} x^{-\mu-3 / 2} \varphi(x) \|_{A} \\
\leq & 2|\mu+k+1| \gamma_{m, k}^{\mu+1}(\varphi)+\gamma_{m+2, k+1}^{\mu+1}(\varphi) .
\end{aligned}
$$

This implies our assertion.

Lemma 4.2. Let $\mu$ and $k$ be as in Lemma 4.1. Then for every $\varphi \in H_{\mu+1}(A)$

$$
h_{\mu, k}\left(M_{\mu} \varphi\right)=x h_{\mu+1, k}(\varphi) \text {. }
$$

Proof. Using the relation

$$
N_{\mu+k-1} \cdots N_{\mu+1} N_{\mu} \varphi(x)=x^{\mu+k+1 / 2}\left(x^{-1} D\right)^{k} x^{-\mu-1 / 2} \varphi(x)
$$

we have

$$
\begin{aligned}
h_{\mu, k}\left(M_{\mu} \varphi\right)= & (-1)^{k} x^{-k} \int_{0}^{\infty} \sqrt{x y} J_{\mu+k}(x y) y^{\mu+k+1 / 2} y^{2}\left(y^{-1} D\right)^{k+1} y^{-\mu-1-1 / 2} \varphi d y \\
& +(-1)^{k} x^{-k}(2 \mu+2 k+2) \\
& \times \int_{0}^{\infty} \sqrt{x y} J_{\mu+k}(x y) \cdot y^{\mu+k+1 / 2}\left(y^{-1} D\right)^{k} y^{-\mu-1 / 2-1} \varphi d y .
\end{aligned}
$$


We now show that $x h_{\mu+1, k}(\varphi)$ reduces to (4.1). Indeed $x h_{\mu+1, k}(\varphi)=(-1)^{k} x^{-k+1} \int_{0}^{\infty} \sqrt{x y} J_{\mu+k+1}(x y) y^{\mu+k+1+1 / 2}\left(y^{-1} D\right)^{k} y^{-\mu-3 / 2} \varphi d y$.

From the formula (see [5])

$$
J_{\mu+k+1}(x y)=-x^{-1} y^{\mu+k} D y^{-\mu-k} J_{\mu+k}(x y)
$$

and an integration by parts, we obtain

$$
\begin{aligned}
x h_{\mu+1, k}(\varphi)= & (-1)^{k+1} x^{-k+1 / 2} \\
& \times \int_{0}^{\infty} y^{2 \mu+2 k+2}\left(y^{-1} D\right)^{k} y^{-\mu-3 / 2} \varphi \cdot D\left[y^{-\mu-k} J_{\mu+k}(x y)\right] d y \\
= & (-1)^{k+1} x^{-k+1 / 2}\left\{\left.y^{\mu+k+2} J_{\mu+k}(x y)\left(y^{-1} D\right)^{k} y^{-\mu-3 / 2} \varphi\right|_{0} ^{\infty}\right. \\
& -\int_{0}^{\infty} y^{-\mu-k} J_{\mu+k}(x y) \\
& \left.\times D\left[y^{2 \mu+2 k+2}\left(y^{-1} D\right)^{k} \cdot y^{-\mu-3 / 2} \varphi\right] d y\right\} .
\end{aligned}
$$

The limit terms vanish because $\varphi \in H_{\mu+1}(A)$. Since

$$
\begin{aligned}
D\left[y^{2 \mu+2 k+2}\left(y^{-1} D\right)^{k} y^{-\mu-3 / 2} \varphi\right]= & y^{2 \mu+2 k+3}\left(y^{-1} D\right)^{k+1} y^{-\mu-3 / 2} \\
& +(2 \mu+2 k+2) \cdot y^{2 \mu+2 k+1}\left(y^{-1} D\right) y^{-\mu-3 / 2} \varphi,
\end{aligned}
$$

we see that $x h_{\mu+1, k}(\varphi)$ equals the right-hand side of (4.1). This completes the proof.

Lemma 4.3. Let $\mu$ be any fixed real number and $k$ a positive integer $\geq-\mu-\frac{1}{2}$. Then, for every $\varphi \in H_{\mu}(A)$,

$$
h_{\mu, k}\left(M_{\mu} N_{\mu} \varphi\right)=-x^{2} h_{\mu, k}(\varphi) .
$$

Proof. From Lemmas 4.1 and 4.2.

Similarly, we can show

Lemma 4.4. Let $\mu$ be any fixed real number and $k$ a positive integer $\geq-\mu-\frac{1}{2}$. Then, for every $\varphi \in H_{\mu}(A)$,

$$
M_{\mu} N_{\mu} h_{\mu, k} \varphi=h_{\mu, k}\left(-x^{2} \varphi\right) .
$$

Theorem 4.1. For any real $\mu$ and $f \in\left[H_{\mu}(A) ; B\right]$,

$$
M_{\mu} N_{\mu} h_{\mu}^{\prime} f=h_{\mu}^{\prime}\left[-x^{2} f\right]
$$

Proof. It follows from Lemma 4.3 that

$$
\begin{aligned}
\left\langle h_{\mu}^{\prime}\left[-x^{2} f\right], \varphi\right\rangle & =\left\langle-x^{2} f, h_{\mu, k} \varphi\right\rangle=\left\langle f,-x^{2} h_{\mu, k} \varphi\right\rangle=\left\langle f, h_{\mu, k}\left(M_{\mu} N_{\mu} \varphi\right)\right\rangle \\
& =\left\langle h_{\mu}^{\prime} f, M_{\mu} N_{\mu} \varphi\right\rangle=\left\langle M_{\mu} N_{\mu} h_{\mu}^{\prime}, \varphi\right\rangle .
\end{aligned}
$$




\section{REFERENCES}

1. A. H. Zemanian, Generalized integral transformations, Interscience, New York, 1968.

2. __ Realizability theory for continuous linear systems, Academic Press, New York, 1972.

3. A. K. Tiwari, Banach space valued distributional Mellin transform and form invariant linear filtering, Indian J. Pure Appl. Math. 20 (1989), 493-504.

4. E. L. Koh, The Hankel transformation of negative order for distributions of rapid growth, SIAM J. Math. Anal. 1 (1970), 322-327.

5. A. Erdélyi, W. Magnus, F. Oberhettinger, and F. G. Tricomi, Higher transcendental functions, Vol. II, McGraw-Hill, New York, 1953.

Department of Mathematics and Statistics, University of Regina, Regina, Canada E-mail address: elkoh@max.cc.uregina.ca

E-mail address: lichen@meena.cc.uregina.ca 